\title{
La producción científica en la Facultad de Lenguas de la Universidad Nacional de Córdoba
}

\author{
SCientific production in the Faculty of Languages at the National University of Cordoba
}

Marta Inés Palacio (martaipalacio@gmail.com) Facultad de Lenguas, Universidad Nacional de Córdoba (Córdoba, Argentina) ORCID: 0000-0002-9757-2237

Sandra Gisela Martín (sandragmartin@gmail.com) Facultad de Filosofía y Humanidades, Universidad Nacional de Córdoba (Córdoba, Argentina) ORCID: 0000-0003-4045-4915

\begin{abstract}
The objectives of this research were, on the one hand, to measure and to quantify the scientific production of teachers and researchers of the School of Languages of the National University of Cordoba between 2008 and 2011; and, on the other hand, to qualitatively analyse and to interpret scientific production within a critical hermeneutical approach. The research was exploratory-descriptive, and was carried out through a mixed methodological approach, quantitative and qualitative. Among the findings, it can be mentioned that the scientific field of the institution is in a moment of self-awareness and reflexivity, that there is a predominance of a professional university model that is in conflict with the production model of scientific knowledge, and that scientific-academic university policies have an impact on the scientific field of the School. There were also several observable issues, namely the academic transformation of the teaching role into teacher-researcher; the absence of a strategic policy of priority areas, the existence of a genuine place for research (infrastructural, academic, economic); the detection of closed groups as "invisible colleges" or "scientific schools" organized around "insular research projects"; and the personalization of the areas and lines of research according to the historical process of the scientific field.
\end{abstract}

Key words: scientific production, publication, scientific knowledge, National University of Cordoba, Faculty of Languages.

\section{Resumen}

El objetivo general de esta investigación fue doble, por un lado, medir y cuantificar la producción científica de docentes e investigadores de la Facultad de Lenguas de la Universidad Nacional de Córdoba del período 2008-2011; y por el otro, analizar e interpretar cualitativamente la producción científica con un abordaje crítico-hermenéutico. La investigación tuvo un carácter exploratorio-descriptivo y se realizó mediante un enfoque metodológico mixto: cuantitativo y cualitativo. Entre los hallazgos encontrados, se puede mencionar que: el campo científico de la Institución se halla en un momento de autoconciencia y reflexividad; existe una predominancia del modelo universitario profesionalista en pugna con el modelo productivo de saber científico; impactan las políticas científico-académicas universitarias sobre el campo científico de la Facultad; se visualiza la difícil transformación académica del rol docente a docenteinvestigador; ausencia de una política estratégica formal de áreas prioritarias; existencia de un espacio 
genuino para la investigación (edilicio, académico, económico); detección de grupos cerrados organizados a modo de "colegios invisibles" o "escuelas científicas", en torno a "proyectos de investigación insulares"; y personalización de las áreas y las líneas de investigación según el proceso histórico del desarrollo del campo científico.

Palabras clave: producción científica, publicaciones, conocimiento científico, Universidad Nacional de Córdoba, Facultad de Lenguas

\section{Introducción: problematizando la producción científica en la universidad}

Reflexionar críticamente sobre la "producción científica" en la universidad supone problematizar aquellos conceptos con los que se la define usualmente y la perspectiva epistemológica que los sostiene.

En la literatura académica hay consenso en definir la producción científica como la creación original de conocimientos pertenecientes a una disciplina determinada, que empleando técnicas, métodos y lenguajes específicos se expone al juicio valorativo de la comunidad científica mediante la publicación de artículos en revistas indexadas, libros, tesis y exposiciones en eventos científicos.

Algunas líneas de metodología científica, muy influidas por modelos bibliométricos positivistas, han restringido la producción científica a propiedades medibles y cuantificables de las publicaciones, obviando que éstas últimas son resultantes de una compleja tarea de la investigación, llegando incluso a reducir la producción científica a un mero conteo numérico de su visibilidad e impacto, ambos definidos por el número de veces en que un texto o artículo científico es citado.

En esta postura, López Yepes define la productividad científica como "la cantidad de investigaciones realizada por los científicos que se aprecia a través de los resultados de la investigación: publicaciones, contribuciones a congresos, patentes y modelos, informes de investigación, entre otros. Existe, además, una delimitación aún más acotada de la producción científica que la circunscribe exclusivamente a aquellas publicaciones aparecidas en revistas científicas indexadas" (2004:339).

Esta perspectiva reduccionista de la producción científica, de cuño positivista, desconoce y desestima los complejos procesos de producción y transferencia del conocimiento, consistentes en múltiples mediaciones sociales y simbólicas, que trascienden el mero acopio numérico de referencias de publicaciones. Además, dicha concepción bibliométrica, en las instancias de justificar la producción científica, fijar las agendas científicas y la asignación de recursos económicos, subestima la importancia del impacto social, entendido éste como las consecuencias directas e indirectas que los desarrollos científico-tecnológicos tienen sobre la sociedad y la ciudadanía.

Hay coincidencia en señalar como una propiedad esencial de la producción científica la nota de originalidad en la elaboración del conocimiento, apreciándose este aspecto desde la lógica particular de cada disciplina: en los métodos, técnicas, conceptos y lenguajes presentados por dicha producción. Se asume que es la propia comunidad científica quien legitima y convalida la producción a través de sus diversos sistemas de evaluación y referatos; ignorándose u ocultándose muchas veces el vicio circular en que cae tal legitimación. A este respecto, según el sociólogo Wallerstein, la ciencia se halla hoy en posición crítica debido a dos razones principalmente. Por un lado, por la poca fiabilidad proveniente de constatar fácticamente modos espurios de legitimación que encubren las reales luchas por el control de los recursos e instituciones de producción del saber que se manifiestan en prácticas académicas extendidas tales como: 
filtros en la información, deformaciones en los mecanismos de evaluación, acciones endogámicas de producción y publicación científica, entre otras (Wallerstein 2005). Por otro lado, y como factor vinculado al anterior, el creciente carácter fragmentario y ultra especializado de la ciencia exige que la validación de la misma quede concentrada en manos de los especialistas prestigiosos de cada disciplina del campo científico, quienes detentan el poder simbólico de instituir los temas consagrados, los métodos y técnicas adecuados, los problemas admisibles $\mathrm{y}$, finalmente, de legitimar argumentativamente el nuevo conocimiento científico producido. Los que poseen mayor capital simbólico en el campo gozan del poder simbólico definido por Bourdieu como "poder de hacer ver y creer" (2003:102).

Por otra parte, la visión cientificista de la producción científica desconoce el contexto social e histórico en la producción del conocimiento científico, estructurado por necesidades y demandas sociales que condicionan la formulación teórica y metodológica de los problemas y objetos de investigación científica (Flax 1998). La distinción que introduce la epistemología y la historia de la ciencia entre historia interna e historia externa de la verdad, permiten visualizar en el origen de las ciencias la matriz cultural y las prácticas sociales que han engendrado los diversos dominios del saber, campos disciplinares con sus objetos, técnicas y métodos, que además han producido a los sujetos de conocimiento (Foucault 2005). Esta perspectiva crítica sobre el conocimiento científico reconoce la producción científica articulada no solo con su contexto socio-histórico, sino también con los sujetos que la producen en un espacio social determinado.

La sociología crítica de Bourdieu aporta elementos que habilitan para una comprensión más compleja de la producción científica. Particularmente la noción de "campo", como "universo en que se incluyen los agentes y las instituciones que producen, reproducen o difunden el arte, la literatura o la ciencia" (Bourdieu 2008b:74), posibilita una indagación y estudio del espacio social del que emana la producción científica. Esta categoría teórica permite un análisis del universo científico como un espacio social relativamente autónomo que, aunque sometido a coacciones externas macrosociales, es poseedor de una lógica propia y de un capital particular -el capital cultural y el capital simbólico-, regido por leyes propias que determinan sus mecanismos y restricciones internas con cierta autonomía de tales presiones externas, autorizando, por lo tanto, un estudio microsocial específico como el pretendido en la presente investigación de estudio de caso.

El planteo bourdiano es dinámico por cuanto concibe al campo científico como un espacio estructurado de relaciones objetivas entre posiciones adquiridas por los agentes científicos, en el que constantemente se producen "luchas epistemológicas" a partir de un tipo específico de relaciones, luchas por el conocimiento y el reconocimiento, es decir, por "el monopolio de la autoridad científica" (Bourdieu 2008b:12). Como dice nuestro autor: "Existir científicamente es distinguirse" (2003:100). Tal distinción se valora según categorías específicas de este espacio social: las contribuciones de los científicos deben aportar conocimientos distintivos, de peso y ser originales. Estos rasgos solo pueden ser apreciados por los "colegas-competidores" (Bourdieu 2003:101) mediante actos de reconocimiento y/o atribución del capital simbólico que les inducirá, luego, a referir y citar la contribución distintiva y original realizada. De aquí que el concepto de "visibilidad" se entienda como "capital simbólico", es decir como reconocimiento del capital científico que posee un determinado científico por su obra o producción otorgado por sus pares de la comunidad científica, y no como mera suma de citaciones. En este espacio social, los juegos y estrategias de lucha en el proceso de producción del conocimiento científico están guiados por el interés de los actores sociales por conservar o adquirir el "capital científico": conocimientos y recursos científicos acumulados (incluidos los recursos financieros), que les posibilite consecuentemente acrecentar el capital simbólico, fama, prestigio o crédito que los demás agentes del propio campo les otorgan. 
Aunque estas luchas por obtener el reconocimiento de una "manera de conocer" -a través del objeto, la teoría y el método- $y$, por ende, conquistar y poseer la "representación legítima de lo real", en cuanto son disputas epistemológicas -aunque no a la suerte de un "todo vale"- se hallan prescriptas y limitadas por las reglas internas de la lógica del propio campo científico: es la propia "comunidad científica" especializada en disciplinas quien finalmente dirime la validez y legitimidad de los nuevos conocimientos mediante formas argumentativas probatorias del valor teórico y peso metodológico de las nuevas contribuciones (Kuhn 1988).

Si bien el campo científico goza de relativa autonomía, el proceso de producción de la ciencia en las universidades se halla actualmente sujeto a condicionamientos político-económicos que ejercen tanto las instituciones del Estado a través de los presupuestos otorgados, como las propias instituciones universitarias de investigación a través de los mecanismos de subsidios e incentivos (Vasen 2013); asimismo, la industria, el mercado y los ámbitos financieros efectúan presiones políticas y económicas sobre la producción científica de las universidades (Rovelli 2015). Particularmente pesa sobre la producción científica universitaria la orientación de la política gubernamental de un país respecto a la ciencia y tecnología. Esta situación de múltiples dependencias plantea tensiones con la pretensión de autonomía de las universidades en la producción de los conocimientos $y$, más aún, conspira con la aspiración del investigador independiente de ejercer libremente la enseñanza e investigación puesto que no solo se halla sujeto a las mismas presiones externas que la institución, sino que además está bajo el dominio de la disciplina que le impone temas y métodos (Vaccarezza 2006).

\section{Marco teórico}

La presente investigación interdisciplinaria, de carácter exploratorio-descriptivo, si bien parte de las definiciones de producción científica como producción original de conocimientos dada por Morales y Morales (1997), intenta superarla al problematizarla epistemológicamente desde una perspectiva crítica, desplazando así la centralidad de los conceptos de raíz positivista y cuantitativa en pos de incorporar otras categorías brindadas por un trabajo descriptivo y crítico-hermenéutico sobre la producción científica que contemple, desde el marco teórico bourdiano asumido, los diversos factores que operan en el campo científico, incluidos sus sujetos o agentes sociales.

Los factores analizados en este estudio de caso, que atraviesan la producción científica de la Facultad de Lenguas en su conjunto, fueron: las políticas científicas explícitas, los condicionamientos institucionales para la investigación, el ejercicio del rol docente-investigador, la orientación de la investigación científica y las áreas prioritarias de la producción científica, la historia de la institución y sus lógicas disciplinares, los debates epistemológicos y teóricos, la demanda e impacto social de las investigaciones.

Este desplazamiento conceptual sobre la producción científica no fue artificial ni forzado, sino que estuvo basado en la "teoría sustantiva" (Sautu 2010:29) desarrollada a lo largo de la investigación; es decir, sobre aquellas proposiciones teóricas elaboradas específicamente durante el proceso investigativo con el fin de estudiar este delimitado espacio social, sus prácticas y efectos materiales. Dicha teoría sustantiva fue construida en un proceso triangular y recursivo durante todo el proceso de la investigación a partir de los datos empíricos obtenidos sobre la producción científica de la Facultad de Lenguas de la UNC del período 2008-2011. De este modo se confrontaron los datos numéricos de la producción científica del período con el marco teórico, mediante un constante proceso conjetural-hermenéutico en confrontación con los datos, el análisis documental del corpus, las observaciones etnográficas de eventos científicos llevados a cabo en 
la Facultad de Lenguas realizadas en 2014-2015 y entrevistas realizadas a "informantes clave" de la institución.

El marco conceptual para el análisis e interpretación de la producción científica de la Facultad de Lenguas se desarrolló a partir de los conceptos de "campo" y "habitus" con los que Bourdieu estudia las prácticas científicas, sin pretender una rígida transferencia conceptual al caso particular estudiado. Esto habilitó explicar y comprender la producción científica como la expresión material y visible de un proceso complejo acontecido dentro de un espacio social determinado, el "campo científico" de la Facultad de Lenguas, en el que se analizó el "habitus" de sus agentes (los docentes-investigadores) constituido por aquellas disposiciones más o menos duraderas incorporadas durante el proceso de socialización en el campo o estructura, produciendo la configuración subjetiva de su sensibilidad, esquemas afectivos y mentalidad. Se asumió la producción científica de la Facultad de Lenguas como un "capital específico", capital científico o capital cultural acumulado constituido por los conocimientos teóricos, métodos, técnicas, instrumentos y textos que circularon en el campo científico y no solo como dato cuantitativo de publicaciones.

El "campo universitario", en un sentido muy amplio, es un campo de poder donde se reproducen los disposicionamientos individuales o colectivos en una relación social de dominante/dominados. Este proceso se lleva a cabo más allá de las voluntades individuales y colectivas ya que se materializa a través del "habitus" de los diversos agentes sociales: alumnos, profesores, autoridades académicas. "El campo universitario reproduce en su estructura el campo del poder cuya estructura contribuye a reproducir por su propia acción de selección e inculcación" (Bourdieu 2008:61).

Sin embargo, el campo universitario no reproduce de modo automático las diferencias sociales o políticas, sino que tiene sus propios "principios de legitimación" que se instituyen en la pugna entre la jerarquización social y política a partir de diversos bienes simbólicos (capital social y político) y la "consagración intelectual" (capital académico: notoriedad académica o científica propiamente dicha). Diferenciar el lugar específico de la Facultad de Lenguas en el campo académico de la UNC excede los fines de esta investigación, pero es un aporte sustancial en la comprensión de los posicionamientos y pugnas que operan entre las facultades, particularmente entre las de las ciencias empírico-analíticas y las ciencias humanas y sociales, por el presupuesto económico para docencia e investigación, entre otras cosas.

Hoy es indiscutible que la producción del conocimiento científico es imprescindible para el desarrollo social y el crecimiento económico; de allí que la innovación sea el eje legitimador de las sociedades del conocimiento que tienden a valorar tanto los conocimientos específicos desarrollados por la tecno-ciencia como los saberes sociales aplicados a la resolución de problemas (Laura Rovelli). Esta perspectiva epistemológica asume el modelo articulador de la tríada: universidad-industria-gobierno, por el que ingresan nuevos agentes y actores en el proceso de definición de las agendas científicas de las universidades (organismos e instituciones internacionales) en las que se configuran las líneas y áreas de investigación a partir de la resolución de problemas, en marcos situados, complejos y pragmáticos, justificando la producción científica por su utilidad social.

No obstante, posiciones epistemológicas críticas señalan ciertos límites éticos y políticos a esta visión pragmática y tecnocrática de la ciencia por cuanto, en su pretendida neutralidad, propende a legitimar socialmente la producción científica y la transformación tecnológica basada meramente en razón del desarrollo económico, bajo el "criterio de eficacia", sin considerar sus consecuencias disfuncionales ecológicas y sociales y/o los efectos indeseados de la automatización de la vida en general (Esther Díaz) generándose nuevos planteos éticos y políticos sobre la ciencia. La tecnocracia, como forma social que 
controla los medios de producción de las sociedades de consumo, se rige por la eficiencia económica y la eficacia del sistema tecnológico. Pretende una neutralidad moral y asepsia ética, a partir de la que considera que las decisiones de los gobiernos se apoyan solo en informes de técnicos y expertos, quienes a su vez acuden a una racionalidad científica para pensar los asuntos, exculpándose así unos a otros de las consecuencias de la tecno-ciencia en una circularidad viciosa.

La postura crítica de la filosofía de la técnica analiza la estructura de la tecno-ciencia y la problematiza como tecnocracia, debatiendo la validación epistemológica pragmática del conocimiento tecno-científico fundada exclusivamente en el "criterio de eficacia" de los conocimientos, inventos y desarrollos tecnológicos; poniendo en la mesa de la discusión las consecuencias disfuncionales para la vida y el planeta (Feyerabend 1987), los impactos sociales y los conflictos éticos suscitados por las innovaciones tecnológicas, la automatización de la vida y por el dominio genético sobre la naturaleza y la sociedad (por ejemplo, el planteado por las biotecnologías). Desde esta posición se acentúa el rol de la ciudadanía en la valoración crítica y legitimación de los conocimientos: los ciudadanos se constituyen en un foro crítico de la ciencia en cuanto son los afectados, beneficiados o perjudicados, por los desarrollos científicostecnológicos.

\section{Antecedentes de estudios sobre la producción científica en la universidad}

Como antecedentes de estudios bibliométricos de la producción científica en universidades se puede mencionar el reciente trabajo sobre la situación en México realizado por Tarango, Hernández-Gutiérrez y Vázquez-Guzmán (2015). Asimismo, el estudio bibliométrico descriptivo de Escobar de Morel y Velázquez de Maldonado (2011) que centró su interpretación en cuestiones de productividad, colaboración y análisis de materias de las investigaciones realizadas en el ámbito de la Universidad Nacional de Asunción, Paraguay, entre los años 2006-2010. Codina-Canet, Olmeda-Gómez y Perianes-Rodríguez (2013) realizaron un análisis de la producción científica y de la especialización temática de la Universidad Politécnica de Valencia, España, en la base de datos Scopus (2003-2010). Maz-Machado, Torralbo-Rodríguez, Vallejo-Ruiz y Bracho-López (2010) confeccionaron un análisis bibliométrico de la producción científica de la Universidad de Málaga en el Social Sciences Citation Index (1998-2007) donde estudiaron la productividad y la distribución temporal, la colaboración nacional e internacional, la producción científica por facultades y las revistas en que se publican los artículos.

Por su parte Picco et al. (2014) estudiaron el fenómeno de la comunicación científica en Uruguay a partir de las publicaciones declaradas en los Currícula Vitae por los investigadores del Sistema Nacional de Investigadores. En una base de datos registraron los datos relativos a las siguientes variables: área de conocimiento, tipo de publicación, tipo de responsabilidad, idioma, lugar de publicación, soporte, arbitraje e indización.

En Argentina, el CAICYT analizó la evolución de la producción científica argentina en Science Citation Index (SCl) entre los años 2000-2004, se analizó y contrastó la producción argentina con el panorama regional latinoamericano. También se analizó la evolución de las publicaciones argentinas en base a distintas variables: áreas disciplinares, niveles de colaboración internacional y patrones de co-publicación con otros países. Posteriormente sobre la base del documento anterior el CAICYT analizó la distribución institucional donde se identificaron las publicaciones de las principales instituciones del sistema científico y tecnológico, particularmente el CONICET y las universidades nacionales. En este estudio se puede apreciar que la UNC se halla entre las 5 instituciones más productivas del país. 
En lo que respecta a estudios bibliométricos sobre universidades argentinas se puede mencionar el trabajo de Centeno (2005) que indagó la producción científica de los proyectos del Programa de Incentivos de la Universidad Nacional de Jujuy (1996-2001) y los trabajos de Miguel (2012; 2012a) que establecieron una serie de indicadores bibliométricos de la Universidad Nacional de La Plata.

A lo largo de su historia, la Universidad Nacional de Córdoba ha desarrollado una extensa tarea de publicación de conocimientos, aunque poco se conoce sobre las características peculiares y cualitativas de su producción científica. Las investigaciones bibliométricas cuantitativas realizadas sobre la UNC (Bustos Argañarás, Centeno Sosa y Rapela 2000; Casarin, Irastorza y Páez 2006; Coronel y Solis 2010; Lanza, Ríos y Rodríguez de Priotti 2013) han afrontado en sus estudios diversas unidades académicas de distintos períodos sin abordar ninguna de ellas estudios sobre la Facultad de Lenguas. Respecto a estudios de enfoque cualitativo, un trabajo pionero ha sido el encarado por Alaniz (2010) que investigó las políticas de investigación en la Escuela de Ciencias de la Información de la UNC en el período 2000-2007, aunque con insuficiente base empírica. El estudio caracterizó las temáticas investigadas en las disciplinas transversales, en particular en las Ciencias de la Comunicación. Indagó la incidencia que ha tenido el modelo de Universidad desde los años '90 y la trama discursiva e institucional neoliberal en la definición de los problemas a investigar.

En cuanto a las áreas prioritarias y/o estratégicas de investigación en universidades nacionales, se debe mencionar el trabajo de Rovelli (2015) que desarrolla un estudio de caso sobre las lógicas de fijación e implementación de las áreas prioritarias de cuatro universidades nacionales (que incluyó entre ellas a la UNC) cotejando las lógicas de investigación de las universidades con las políticas de organismos e instituciones científicas nacionales sobre áreas prioritarias. Entre sus conclusiones afirma que las universidades más tradicionales con gestión científica centralizada, como lo es la UNC, la política de autonomía hace que los grupos de investigación consolidados sean los que moldeen las agendas temáticas y las áreas científicas según sus propios intereses, provocando dispersión y superposición de recursos, como también una fragmentación disciplinar del conocimiento; situación observada en la presente investigación.

En lo que respecta al nuevo perfil del docente-investigador, el trabajo de Coiçaud (2008) presenta un estudio cualitativo sobre la actividad investigativa en la universidad donde indaga las voces de los investigadores, las vivencias y los propósitos, las dificultades, las expectativas y las dudas. La investigación abordó las prácticas y estrategias de supervivencia de los profesores investigadores con un enfoque interpretativo acerca de las actividades profesionales de docentes que enseñan e investigan, señalando las dificultades en el proceso de reconversión del rol docente a docente-investigador exigido por la Ley de Educación Superior de 1995.

Se puede mencionar, como antecedente relevante del tipo de investigación mixta sobre investigación y producción científica, al trabajo de Escotet, Aiello y Sheepshanks (2010), el que da cuenta de la actividad científica de los investigadores de la universidad argentina en el contexto de América Latina. A través de una prolija y exhaustiva recolección de datos cuantitativos y cualitativos sobre los investigadores (cantidad, cargos, dedicación, concursos, programas de subsidios y financiamiento, gastos en investigación y desarrollo según disciplinas, número de alumnos, situación y tendencia de los posgrados) establece que la situación de precariedad de los investigadores en Argentina se mantiene pese a que en un primer momento pareciera haber mejorado por la inversión estatal. Otros investigadores en un informe sobre el estado de la ciencia en Iberoamérica publicado por RICYT (red de indicadores de ciencia y tecnología iberoamericana e interamericana) confirman que no hay una relación directa entre inversión y producción 
científica, lo cual indicaría que las causas del crecimiento de la producción científica depende de factores más complejos y variados como son la vocación científica, la mentalidad, las disposiciones y actitudes de los agentes universitarios que han debido reconvertirse en docentes-investigadores en los últimos años.

Asimismo, constituye un antecedente valioso la investigación cualitativa de Naidorf y Pérez Mora (2012) sobre las condiciones de producción intelectual en Argentina, Brasil y México en que problematizan y analizan comparativamente las políticas científicas nacionales y universitarias que afectaron, a partir de la década del ‘90, las condiciones de producción intelectual en dichos países debido a los cambios sociales, políticos y económicos a nivel local y mundial.

\section{Metodología de la investigación}

El primer estudio mixto (cuantitativo y cualitativo) sobre la producción científica en la Facultad de Lenguas ha sido llevado a cabo por la presente investigación documental crítico-hermenéutica. El objetivo de la investigación fue estudiar y comprender la dinámica y tendencia de las investigaciones de la Facultad de Lenguas de la UNC en su contexto histórico, a partir del análisis de los datos numéricos de su producción científica (2008-2011) y su interpretación en función de ciertas propiedades cualitativas y factores del campo científico en el cual se produjo y del habitus de los agentes: condiciones de producción, políticas científicas asumidas por la gestión, posiciones ideológicas y epistemológicas en el área de las ciencias y estudios del lenguaje, opciones metodológicas exhibidas por los artículos científicos (elección de fuentes para publicar y los idiomas de las publicaciones), temáticas, áreas y líneas disciplinarias desarrolladas en las investigaciones y en la producción científica en general, demandas sociales a las que atienden y el impacto social de la producción científica de la Facultad de Lenguas.

En el trabajo de investigación se cuantificó la producción científica del 2008-2011 en su variedad documental tipológica (libro, capítulo de libro, traducción de libro, manual de cátedra/ciclo nivelación, artículo científico, artículo de divulgación, artículo en periódico, revista/presentación/prólogo, reseña, ponencia nacional/internacional, panel, tesis) y se indagó sobre propiedades cualitativas de la producción científica, tales como: áreas y líneas temáticas principales, recursos materiales y humanos, condiciones y factores institucionales, políticas de investigación de la Facultad de Lenguas, rol del docente-investigador, demandas sociales e impacto social, debates teóricos y epistemológicos sobre las ciencias del lenguaje.

El estudio cualitativo incluyó un análisis textual-documental de una muestra seleccionada del corpus: los 50 artículos científicos publicados en el período. Se analizaron solamente los artículos científicos del período en base a variables: a) teóricas: paradigma, líneas, áreas y corrientes teóricas; y b) epistemológicas-metodológicas: objetivos, tipo de investigación, palabras clave, enfoque, corpus/población/muestra, instrumentos, conclusiones, impacto social. Luego, estos resultados cualitativos se interpretaron en triangulación con las propiedades obtenidas de registros etnográficos abiertos realizados en eventos científicos acontecidos en el campo y entrevistas semi-estructuradas realizadas a informantes clave de la institución seleccionados por su rol de investigación o de gestión de la política de ciencia y técnica de la unidad académica.

Por los datos obtenidos, el análisis bibliométrico y la interpretación cualitativa de la producción científica realizada, este trabajo de investigación se constituye en precursor de otras investigaciones sobre el desarrollo interno de la base científica de la Facultad de Lenguas y de la UNC. Con la presente investigación se aportan elementos semánticos y valorativos-hermenéuticos a los indicadores bibliométricos obtenidos, ampliando los sentidos de la definición de la producción científica a partir de la construcción de una teoría 
sustantiva; proceso imprescindible a los efectos de diagnosticar y comprender integralmente el caso de la producción científica de la Facultad de Lenguas.

\section{Resultados y consideraciones finales: interpretación de la producción científica de la Facultad de Lenguas}

Las conclusiones de esta investigación tienen la marca de orilla de todo conocimiento científico: son provisorias. Han sido elaboradas reflexivamente a la luz del marco teórico asumido sobre el conocimiento y la producción científica, de la labor crítico- hermenéutica de los datos bibliométricos sistematizados en la Facultad de Lenguas (2008-2011), del análisis documental de los artículos científicos (muestra) y de los registros etnográficos de las observaciones y entrevistas realizadas a los informantes clave. Sobre las expresiones de éstos últimos, quienes como actores sociales del espacio social cargan sobre sí una interpretación o "primera hermenéutica" de los sentidos de sus acciones y prácticas, se realizó una "segunda hermenéutica".

Sin pretender realizar una enumeración exhaustiva ni una comprensión total del fenómeno de la investigación y la producción científica en la Facultad de Lenguas, que probablemente será modificada con los datos que se obtengan del estudio del período 2012-2015 actualmente en curso, la investigación realizada hasta aquí permite señalar algunos ejes interpretativos:

1. Se advierte que el campo científico de la Institución se halla en un momento de autoconciencia y reflexividad patentizado en la necesidad de diagnosticar y evaluar la producción científica y la investigación en general a los fines de establecer acciones para fortalecer y desarrollar la investigación. Esto coincide con la finalidad del convenio realizado con el Ministerio de Ciencia, Tecnología e Innovación Productiva de la Nación (MINCYT) para implementar el proyecto de autoevaluación para las universidades públicas (PEI) puesto en marcha en 2015.

2. Predominancia del modelo universitario profesionalista en pugna con el modelo productivo de saber científico bajo el que se van reformando en los últimos años las universidades del país. En la Facultad de Lenguas esta supremacía profesionalista aún puede observarse en el énfasis de los roles de traducción profesional y docencia de las lenguas por encima del de la producción científica de conocimientos teóricos o aplicados sobre el lenguaje y las lenguas. Esta primacía profesionalista se verifica en la matrícula que tienen los traductorados y profesorados en relación con las licenciaturas y posgrados. Esta propiedad también se observa en el habitus de los docentes construido en el espacio institucional; habitus que exhibe esquemas mentales y perceptivos organizados por representaciones de sí mismos bajo el rol docente prioritariamente. Esta sería una clave interpretativa del volumen y tipo de la producción científica recogida en el período.

Esta situación observada y analizada en el campo científico de la Facultad de Lenguas probablemente obedezca al mandato fundacional de la institución surgida en el año 1920 para enseñar las lenguas, respondiendo así a una demanda social de la que la universidad se hizo cargo habilitando carreras profesionales que otorgaran los títulos de profesores de español y otros idiomas requeridos por el sistema educativo (particularmente el nivel medio, terciario y universitario).

Los orígenes de la Facultad de Lenguas se remontan al año 1920 al denominado Departamento de Idiomas que dependía de la Facultad de Derecho de la UNC y que enseñaba, en ese entonces, francés, italiano y nociones básicas de latín jurídico. Con el correr del tiempo se transformó en 1926 en Instituto de Idiomas 
y sus planes de estudio se fueron modificando hasta llegar a incluir asignaturas de carácter didácticopedagógico en el Profesorado y de carácter técnico en el Traductorado. Posteriormente, en 1943 se transformó en Escuela Superior de Lenguas, dependiente de Rectorado, otorgando títulos de grado, y el 5 de Agosto de 2000, luego de muchos años de reconocida trayectoria, se convirtió en Facultad de Lenguas al ser aprobada su creación por la Asamblea Universitaria. En la actualidad se dictan profesorados, traductorados y licenciaturas en seis idiomas: inglés, italiano, francés, alemán, portugués y español, este último orientado hacia su enseñanza como lengua materna y extranjera y se expenden varias titulaciones de posgrado (especialidades, maestrías y doctorado) relacionados a las ciencias del lenguaje y las lenguas.

Más allá de la penetración y longevidad del modelo profesionalista, característica compartida con otras unidades académicas y el sistema universitario en general que aún muestra resabios del modelo de universidad napoleónico (Escotet et. al. 2010), en el campo científico de la Facultad de Lenguas emerge otro elemento comprensivo de la pregnancia del modelo profesionalista: el habitus de los sujetos, que determina su propia autocomprensión identitaria a partir de la conformación histórica del rol docente por encima del de investigador. Estos elementos explicarían el grado de desarrollo científico de la Facultad de Lenguas exhibido en la producción científica, y también la preocupación de la gestión por transformar ciertas reglas internas del campo a fin de incentivar y fortalecer la investigación en su seno a través de implementar una paulatina política de investigación con acciones como la llevada a cabo por el "Programa de Formación de Investigadores", instrumentado por la gestión a partir del año 2015, por el que la Facultad otorga subsidios para la investigación con recursos propios a equipos constituidos por docentes, alumnos y egresados a los fines de favorecer su inserción en la investigación y dirigidos por aquellos docentes que no han logrado ingresar al régimen de categorización y/o incentivos del MINCyT o cuyas categorías no le permiten aún dirigir proyectos.

3. Impacto de las políticas científico-académicas universitaria sobre el campo científico de la Facultad de Lenguas. La transformación académica del rol docente a docente-investigador que se sustancia jurídicamente en el año 2008 produce un corte cronológico, un antes y un después de la investigación en general, especialmente en la Facultad de Lenguas. La creación y organización de la Carrera Docente por los Estatutos de la UNC, que modificó las reglas del campo académico de la Universidad, parece haber sido un proceso complejo que afectó el status quo de varios agentes sociales en el campo científico de la Facultad de Lenguas y supuso aprendizajes graduales y desiguales de las estrategias de investigación y producción científica para las que pocos se hallaban preparados, además de un cambio profundo en las subjetividades y habitus de los actores sociales. Podría decirse que históricamente en la Facultad de Lenguas hubo dos etapas en la conformación del habitus distinguibles en función de un cambio fundamental en la estructura del campo: la creación de la Facultad en el año 2000. Un primer momento, previo a la constitución de la Facultad, en el que los docentes formados en investigación tenían mayoritariamente trayectorias personales en las letras y estudios literarios, aunque no formalizados a nivel local en los sistemas de SECYT, ni de categorización e incentivos a nivel nacional. Los rasgos predominantes de este grupo de agentes era la orientación a la divulgación científica, más que a la producción y publicación en revistas indexadas; la identidad conformada bajo el rol docente; el posgrado o el título de doctorado concebido como la culminación de la carrera académica y no como el inicio o la habilitación de la misma.

Un segundo momento, ya aludido, que podría situarse entre los años 2003-2004 en el que ingresan docentes con trayectoria consolidada en investigación y conjuntamente con aquellos que ya se hallaban en la institución pero que lograron transformarse, obtener titulaciones de posgrado en otras unidades 
académicas y/o universidades; en esta etapa se produjo el crecimiento cuantitativo y cualitativo de la producción científica.

Tanto en la recolección de datos, en las entrevistas y observaciones realizadas, como en el análisis del corpus se detectaron diversas problemáticas que develan la matriz de una cultura institucional regida por una lógica vinculada más a la docencia que a la investigación. Sin embargo, hay indicios que muestran un cambio en las reglas internas del campo científico y las posiciones en el mismo, como también una transformación en el habitus de los agentes de la Facultad (docentes investigadores). El cambio estructural fue dinamizado por la necesaria renovación de la planta docente a través de los concursos docentes y el ingreso a la Carrera Docente de los agentes que tenían sus concursos vigentes al año 2008; ambos procedimientos institucionales se tornaron imprescindibles para legitimar sus posiciones en el campo académico. Los ejes evaluatorios del desempeño docente incluirán explícitamente a la investigación, articulada con la docencia, la extensión y la gestión universitaria.

En este contexto de transformación del campo académico en razón de las políticas universitarias, emerge la investigación como un elemento simbólicamente distintivo, la cual deja de ser algo circunstancial o reservado a quienes poseen genuino interés científico, sino que se concibe como actividad imprescindible para acceder y mantenerse en los cargos docentes, quedando investida de estatus. La consolidación de la carrera docente trajo aparejado la aparición de un nuevo perfil de los actores sociales: el docenteinvestigador. Con este nuevo rol bifronte se intensificó el trabajo académico e incrementó notablemente la cantidad de tareas y funciones que deben asumir los profesores-investigadores en el seno de sus instituciones: presentación de proyectos, elaboración de presupuestos, presentación de informes, asunción de cargos de gestión, participación en tribunales de concursos, dirección de tesistas, realización de tareas de asesoramiento, evaluación de proyectos e informes, coordinación de propuestas editoriales.

Asimismo, es un indicativo del cambio en el espacio estructural del campo y el habitus de la Facultad de Lenguas la incorporación de nuevas generaciones de docentes con un habitus ya transformado: perfiles de posgrado inmediatamente obtenidos luego del grado, conocimientos y habilidades en prácticas en investigación y comunicación científica, experiencia en trabajos interdisciplinarios, entre otros.

4. Al historiar el proceso de inicio y desarrollo de la investigación en la Facultad de Lenguas los datos obtenidos sobre la producción científica se contextualizan y ubican en una interpretación que los integra con la autorreflexión de sus propios sujetos.

Los relatos de los actores sociales entrevistados presentan interesantes matices del proceso histórico de reconversión del rol docente al de investigador, los que indicarían que la investigación en la Facultad de Lenguas fue percibida en décadas anteriores como "campos alambrados" solo accesibles para investigadores de las agencias científicas nacionales y provinciales, pero no para la masa docente en general [entrevista 2]. En este sentido, hay coincidencias en señalar que el desarrollo de la investigación en las universidades fomentado por las políticas estatales del MINCyT y la Secretaría de Políticas Universitarias (SPU) -como el Régimen de Incentivos a los investigadores- junto con la transformación instituida por los Estatutos de la UNC de 2008, fue un proceso gradual, lento y dificultoso, tanto para ingresar en el campo de la investigación, categorizarse para entrar en el sistema de incentivos y poder conformar equipos de investigación (como integrantes y/o directores), acceder a los subsidios y obtener información sobre becas y programas. En este sentido en la Facultad de Lenguas el rol de investigador se presentó como un "territorio para la conquista y hubo que conquistarlo" [entrevista 2]. 
5. Ausencia formal de una política estratégica de áreas prioritarias pese a que desde la gestión y diversos actores sociales de la institución se aspira a promover ejes de investigación en concordancia con las políticas científicas de la UNC, acoplándose a los ejes de la gestión centralizada de la universidad: interculturalidad, plurilingüismo e interdisciplinariedad. En el análisis del corpus y la producción científica se observa que estos ejes aún no han impregnado los proyectos de investigación y la producción científica del campo en su conjunto. Estas áreas prioritarias no se reflejan en la producción científica del período, la cual exhibe una alta fragmentación, insularidad y dispersión temática en concordancia con la característica general de la investigación de la Universidad Nacional de Córdoba señalada por la investigación de Rovelli (2015).

En las entrevistas, los actores sociales reconocen la existencia de áreas de vacancia en la investigación, lo cual puede verificarse en la producción científica del período, tales como: insuficiente desarrollo de los estudios sobre la lengua en relación con otros campos disciplinares: concepción de la lengua según los procesos históricos, polémicas en torno a la lengua, reflexión meta-lingüística, relación de los estudios del lenguaje y la lengua con los planteos filosóficos del giro lingüístico, estudios conceptuales de las palabras y etimologías, vinculación de la lengua con la literatura y otras áreas de la cultura, etc.

De los registros etnográficos en relación a áreas y líneas epistemológicas, se constata que en el período han surgido incipientes investigaciones y estudios de ecocrítica, con planteos interdisciplinarios novedosos, que convocan particularmente a docentes-investigadores de la sección de lengua inglesa de la Facultad de Lenguas debido al contexto originario angloparlante de tales estudios.

Del análisis documental del corpus se desprende una baja densidad de la producción científica en el área de lingüística teórica, si se compara con la producción de investigaciones literarias y enseñanza de las lenguas. Luego del análisis de los artículos científicos, se advierte que hay áreas deprimidas; insuficientes desarrollos en ciertas líneas y áreas, tales como neurolingüística, psicolingüística, sociolingüística; pragmática y filosofía del lenguaje.

La situación descripta y analizada puede interpretarse como un proceso de reproducción social de la lógica institucional reguladora del campo científico de la Facultad de Lengua, que se halla internalizada en el habitus de los agentes. Esta lógica funcionaría como un "obstáculo epistemológico" para nuevas conceptualizaciones, paradigmas y formulación de temáticas interdisciplinarias, además de constituir un límite político para implementar un plan estratégico de áreas prioritarias para la Facultad de Lenguas en función de los conflictos y tensiones que se pudieran originar entre los actores sociales. "La maniobra de propiciar áreas es una maniobra difícil desde el punto de vista político, porque significaría otorgar prioridades a determinados temas y áreas, y eso podría determinar una serie de conflictos en algún momento" [entrevista 2].

Esto señalaría la necesidad de priorizar áreas y reordenar los proyectos de investigación, redefiniendo las prioridades institucionales en función del anclaje en la realidad socio-histórica. Por lo mismo, las líneas de investigación y producción científica de áreas prioritarias para la Facultad deberían establecerse desde una definición de políticas científicas sobre el lenguaje y las lenguas "pensando en las agendas teóricas que circulan en lo que respecta a lengua su consustanciación indisoluble con la cultura y con la problemática que se genera a nivel mundial (...) con el área de políticas lingüísticas y con lo que hace a los ejes de interculturalidad, plurilingüismo y estas cuestiones de (...) eso me parece que tendrían que ser, por lo menos desde mi modesto entender, los ejes prioritarios. Por supuesto los enfoques interdisciplinarios, es 
decir, la lengua pensada en consustanciación con la cultura, y atravesando todas las disciplinas" [entrevista 6].

También la definición de las áreas requiere del diseño de una "lógica concentrada" (Rovelli 2015:43) de orientación de la investigación científica, con acciones que involucren a todos los actores que se hallan en el campo: quienes gestionan (autoridades de la Facultad de Lenguas y Secretaría de Ciencia y Técnica), los investigadores, el Centro de investigaciones de la Facultad de Lenguas (CIFAL) y los posgrados (docentes y estudiantes de posgrado).

6. De las observaciones, registros etnográficos y entrevistas realizadas en el año 2015, se desprende que en la Facultad de Lenguas en los últimos años se ha venido construyendo paulatinamente un espacio genuino para la investigación (edilicio, académico, económico) con estrategias de diversos efectos y resultados: organización y ejecución de numerosos eventos científicos, algunos de ellos superpuestos entre sí produciendo cierta saturación de actividades y dispersión de los debates; alta proporción de publicaciones de ponencias en congresos y jornadas en comparación con publicaciones de artículos científicos; publicaciones endogámicas (actas de congreso y libros) con insuficiente impacto en debates transfronterizos a los grupos y disciplinas; monolingüismo mayoritario en las publicaciones, siendo el español la lengua en que se publica la mayoría de los trabajos; prestigio académico y científico concentrado en algunas individualidades o grupos; baja articulación entre investigación y posgrados (líneas, áreas, problemas, integrantes).

De estos registros del 2015 se observó sobre-oferta de eventos en el mismo año académico y espacio institucional; baja asistencia de personas externas a la Facultad de Lenguas (excepto los invitados y los interesados directos) y poca asistencia de estudiantes, egresados y docentes en general; fragmentación de públicos: la presencia y apoyo de grupos de investigadores y académicos identificados con los organizadores y/o líneas temáticas desarrolladas en los eventos; desconocimiento del resto de los miembros de la institución sobre las actividades realizadas en tales reuniones científicas. En los congresos y las ponencias predominaron conductas endogámicas y de grupos cerrados que se contrapone con la publicación de los 50 artículos científicos del período investigado donde el $70 \%$ de los artículos se publicó en el exterior. En cambio, en la publicación de libros hubo un mayor equilibrio ya que la mitad fue interna a la institución (el $50 \%$ se publicó dentro de Facultad de Lenguas o UNC).

7. Se detectan grupos cerrados a modo de "colegios invisibles" o "escuelas científicas" organizadas en torno a "proyectos de investigación insulares" [entrevista 1], reproduciéndose las divisiones de cátedras y secciones de las lenguas del campo institucional académico en el campo científico. Se observa la reproducción social e institucional circular de las separaciones de las "escuelas" de investigación, transfiriéndose en los procesos de enseñanza-aprendizaje y de éstos a la investigación: los alumnos y docentes aprenden esta cultura institucional y la reproducen. Esto se refleja en los congresos (temática, participación, ponencias) y en la producción científica en general: se manifiestan así fronteras epistemológicas internas entre las diversas áreas de estudios del lenguaje que obturan la fluidez en los tránsitos teóricos, trasmigraciones e intercambios de perspectivas, conceptos y problemas. Se observa falta de comunicación y debates entre los grupos y equipos de la institución: situación percibida en diversos congresos y jornadas (registros 2015) donde la ausencia de miembros e investigadores de otras áreas del campo es ostensiva.

8. La situación de la investigación y de la producción científica del campo científico devela una personalización de las áreas y las líneas de investigación según el proceso histórico. Las iniciativas 
personales fueron las que marcaron la constitución de las áreas del CIFAL, los proyectos de investigación, las publicaciones y la actividad científica en general. La constitución del campo científico la Facultad de Lenguas estuvo signado por la historia interna: grupos y áreas, y la historia externa: condicionamientos socio-políticos y coacciones de la universidad, y no tanto la lógica de las disciplinas del lenguaje y sus debates epistemológicos. Los límites y fronteras entre los estudios sobre el lenguaje y la lengua estuvieron dados por la historia externa: acuerdos coyunturales y políticos; y por la personalización de las cátedras y grupos.

En este contexto hay que señalar el peso de decisiones políticas académicas de la UNC sobre la producción científica de la Facultad de Lenguas, tales como la separación disyuntiva entre Lengua y Literatura, con su correspondiente demarcación de los terrenos disciplinares: el estudio lingüístico y de la enseñanza de las lenguas a la Facultad de Lenguas, y los estudios literarios y enseñanza de la literatura en la Escuela de Letras (dependiente de la Facultad de Filosofía y Humanidades). A través de acuerdos institucionales se fijó como competencia profesionalista para los egresados de las carreras de la Facultad de Lenguas la enseñanza de las lenguas del Ciclo Básico del Nivel Medio; y para la competencia profesionalista para los egresados de la carrera de Letras (Facultad de Filosofía y Humanidades) la enseñanza del ciclo de Especialización de dicho nivel educativo.

Tales acuerdos históricos exhiben un interesante nivel de forzamiento, ya que por el afán de lograr cierto equilibrio en el reparto de los saberes y para prevenir solapamientos, competencias profesionales, luchas epistemológicas y de autoridades simbólicas entre las dos instituciones de la UNC, no se reparó en que sus estudios se hallan emparentados de modo inescindible por el tema común del lenguaje: el sistema de reglas de la lengua se expresa en el lenguaje y sus documentos orales y escritos.

Así el proceso histórico-político de institución de los saberes en la UNC tomó la deriva de creación de dos campos académicos separados: Facultad de Lenguas, por un lado, y Escuela de Letras, por el otro, con un campo epistémico común constituido por objetos que, aunque conservando sus matices, enfoques y perspectivas disciplinarias, se implican recíprocamente y entrecruzan constantemente por cuanto los estudios de la lengua debieran atender los estudios de las obras literarias en que la lengua se materializa y viceversa.

Esta situación se refleja en el corpus de artículos científicos del período cuya mayor proporción corresponde a producciones de literaturas comparadas. Es llamativo que las revistas científicas de mayor publicación sean las destinadas a estudios y análisis literarios, lo cual puede comprenderse como parte del proceso histórico de conformación de la masa crítica de la Institución, donde un gran número de docentes con formación de posgrado han provenido en los últimos años de la carrera de Letras, que se imparte en la Facultad de Filosofía y Humanidades, por no existir títulos de posgrado con anterioridad a 2000 en que se crea la Facultad de Lenguas.

Se constata que actualmente son varios los docentes-investigadores que trabajan simultáneamente en la Facultad de Lenguas y en la Escuela de Letras de la Facultad de Filosofía y Humanidades, integrando proyectos de investigación radicados en una u otra institución. A partir de los años 2003-2004 se produce un salto cualitativo en investigación en la Facultad de Lenguas debido a la incorporación por el sistema de concursos de profesores con trayectoria consolidada en investigación que dinamiza la implementación de proyectos. 
9. La presente investigación contribuye al diagnóstico de la producción científica en la Facultad de Lenguas y aporta a la gestión elementos para la revisión y formulación de áreas prioritarias de investigación que articulen los posgrados con las investigaciones que se desarrollan en el grado. En la medida en que se difundan y discutan los datos y las conclusiones se contribuirá a los procesos de revisión y cambio de los planes de estudios, actuales o futuros.

Con el presente trabajo, y su actual continuación, se aporta una sólida investigación cuantitativa y cualitativa imprescindible para un riguroso diagnóstico del estado de la producción científica de los últimos años de la Facultad de Lenguas de la Universidad Nacional de Córdoba en vistas a generar información fiable que brindar elementos y criterios para la orientación y evaluación de las actividades científicas y tecnológicas de la unidad académica, en concordancia con la intencionalidad del Programa de Evaluación de Investigación (PEI). También brinda información estratégica para la y toma de decisiones en políticas científicas de la unidad académica estudiada.

\section{Nota}

Este artículo corresponde a una investigación realizada sobre la producción científica de la Facultad de Lenguas de la Universidad Nacional de Córdoba (2008-2011), dirigida por Marta Inés Palacio, codirigida por Sandra Gisela Martín e integrada por Mercedes Abriola, María Inés Altamirano, Sergio Augusto Navarro y Nicolás Rizzi. Esta investigación fue avalada y financiada por la Secretaría de Ciencia y Tecnología (SECYT) de la UNC. Su segunda etapa (2012-2015) se haya actualmente en curso.

\section{Bibliografía}

Alaniz, M. 2010. Políticas de investigación en una unidad académica de la Universidad Nacional de Córdoba (2000-2007). Cuadernos del Instituto Antonio de Nebrija 13: 13-26. http://erevistas.uc3m.es/index.php/CIAN/article/download/1/1

Bourdieu, P. 2003. El oficio del científico. Barcelona: Anagrama.

Bourdieu, P. 2008. Homo academicus. Buenos Aires: Siglo Veintiuno Editores.

Bourdieu, P. 2008b. Los usos sociales de la ciencia. Buenos Aires: Nueva Visión.

Bustos Argañaraz, E; Centeno Sosa, A. y Rapela, M.V. 2000. Producción científica de los investigadores con proyectos aprobados por la Secretaría de Ciencia y Tecnología de la Universidad Nacional de Córdoba, 1996/1999: análisis bibliométrico (Tesis de licenciatura). Córdoba: Universidad Nacional de Córdoba.

Casarín, M; Irastorza, R. y Páez, F. 2006. La difusión científica en la Universidad Nacional de Córdoba. Las publicaciones periódicas en el Quinquenio 2000-2004. Astrolario 3.

http://www.astrolabio.unc.edu.ar/articulos/publicacionescientificas/articulos/casarin-irastorza-paez.php

Centeno, M.E. 2005. La producción científica de los proyectos del Programa de Incentivos de la Universidad Nacional de Jujuy (1996-2001): un estudio bibliométrico (Tesis de licenciatura). Córdoba: Universidad Nacional de Córdoba. http://eprints.rclis.org/11097/ 
Codina-Canet, M.A.; Olmeda-Gómez, C. y Perianes-Rodríguez, A. 2013. Análisis de la producción científica y de la especialización temática de la Universidad Politécnica de Valencia. Scopus (2003-2010). Revista Española de Documentación Científica 36(3): e019. doi: 10.3989/redc.2013.3.942

Coiçaud, S. 2008. El docente investigador. La investigación y su enseñanza en las universidades. Madrid: Miño y Dávila.

Coronel, N. B. y Solís, D. 2010. Grado de adecuación de las revistas de la Universidad Nacional de Córdoba a las normas internacionales de presentación, período 2000-2007. (Tesis de licenciatura). Córdoba: Universidad Nacional de Córdoba.

Escobar de Morel, M. y Velázquez de Maldonado, H. 2011. Productividad científica en la Universidad Nacional de Asunción: estudio bibliométrico descriptivo 2006-2010. Asunción: Universidad Nacional de Asunción.

http://www.pol.una.py/sites/default/files/files/Articulo_cientifico_Productividad_cientifica_UNA.pdf

Escotet, M.A; Aiello, M. y Sheepsahnks, V. 2010. La actividad científica en la Universidad. Buenos Aires: Universidad de Palermo.

Flax, J. 1998. La historia de la ciencia: sus motores, sus frenos, sus cambios, su dirección. (pp. 43-55). En:

E. Díaz. La ciencia y el imaginario social. Buenos Aires: Biblós.

Feyerabend, P. 1987. Adiós a la razón. Buenos Aires: Rei Argentina.

Foucault, M. 2005. La verdad y las formas jurídicas. Barcelona: Gedisa.

Kuhn, T. 1988. La estructura de las revoluciones científicas. Buenos Aires: Fondo de Cultura Económica.

Lanza, J.M; Ríos, M.M. y Rodríguez de Priotti, I.R. 2013. Estudio bibliométrico de artículos científicos producidos en la Facultad de Ciencias Químicas de la Universidad Nacional de Córdoba desde 2000 a 2009. (Tesis de licenciatura). Córdoba: Universidad Nacional de Córdoba.

López Yepes, J. 2004. Diccionario enciclopédico de ciencias de la documentación. Madrid: Síntesis.

Maz-Machado, A; Torralbo-Rodríguez, M; Vallejo-Ruiz, M. y Bracho-López, R. 2010. Análisis bibliométrico de la producción científica de la Universidad de Málaga en el Social Sciences Citation Index (1998-2007). Revista Española de Documentación Científica 33(4): 582-599.

http://redc.revistas.csic.es/index.php/redc/article/viewArticle/672

Miguel, S. 2012. Producción científica de la UNLP en revistas indizadas en Web of Science (WoS) y SCOPUS, 2006-2010. La Plata: Universidad Nacional de La Plata.

Miguel, S. (Coord.) 2012a. Serie Indicadores Bibliométricos de La UNLP. La Plata: Universidad Nacional de La Plata.

Morales, M. y Morales, M. 1997. La informetría y las fuentes de información personales e institucionales. Su importancia en relación con la información de inteligencia. Ciencias de la información 28(3): 207-217. 
Naidorf, J. y Pérez Mora, R. (Coord). 2012. Las condiciones de producción intelectual en Argentina, Brasil y México. Buenos Aires: Miño y Dávila.

Picco, P; Aguirre-Liguera, N; Maldini, J; Simón, L; Petroccelli, P; Fontans, E; Fager, J. C. y Ceretta, G. 2014. La comunicación científica en Uruguay: estudio de las publicaciones de los investigadores activos del Sistema Nacional de Investigadores (2009-2010). Transinformação 26(2): 155-165. doi: 10.1590/010337862014000200005

Rovelli, L.I. 2015. Un modelo para armar: áreas prioritarias e investigación en universidades nacionales. Ciencia, Docencia y Tecnología 26(51): 26-53.

http://www.produccióncientíficaient.uner.edu.ar/index.php/cdyt/article/view/112/142

Sautu, R. 2010. Manual de metodología. Buenos Aires: CLACSO-Prometeo.

Tarango, J; Hernández-Gutiérrez, P.Z. y Vázquez-Guzmán, D. 2015. Evaluación de la producción científica en universidades públicas estatales mexicanas (2007-2011) usando análisis de componentes principales. El Profesional de la Información 24(5): 567-576.

http://www.elprofesionaldelainformacion.com/contenidos/2015/sep/06_esp.pdf

Vaccarezza, L. 2006. Autonomía universitaria, reformas y transformación social, pp. 33-49. En: H. Vessuri. Universidad e investigación científica. Buenos Aires: CLACSO.

Vasen, F. 2013. Las políticas científicas de las universidades en el sistema científico nacional. Ciencia, Docencia y Tecnología 24(46): 9-32.

http://www.revistacdyt.uner.edu.ar/spanish/cdt_46/documentos/46_dossier01_vasen.pdf

Wallerstein, I. 2005. Las incertidumbres del saber. Barcelona: Gedisa.

Recibido el 1 Jun 2016

Aceptado el $18 \mathrm{Jul} 2016$ 